\section{US LI-RADS visualization score: diagnostic outcome of ultrasound-guided focal hepatic lesion biopsy in patients at risk for hepatocellular carcinoma}

\author{
Ji Hun Kang*, Sang Hyun Choi, So Yeon Kim, So Jung Lee, Yong Moon Shin, \\ Hyung Jin Won, Pyo-Nyun Kim
}

Department of Radiology and Research Institute of Radiology, Asan Medical Center, University of Ulsan College of Medicine, Seoul, Korea

Purpose: This study investigated the diagnostic outcome of ultrasound (US)-guided focal hepatic lesion biopsy in patients at risk for hepatocellular carcinoma (HCC) and evaluated the US visualization score as a risk factor for non-diagnostic results.

Methods: We retrospectively evaluated 208 focal hepatic lesions in 208 patients who underwent US-guided biopsy in 2016. Using the US Liver Imaging Reporting and Data System version 2017, each exam was assigned a US visualization score (A, B, or C). Final diagnoses were made using pathology reports, and biopsy results were categorized as diagnostic or non-diagnostic. Univariable and multivariable analyses were performed to determine risk factors for nondiagnostic results, including US visualization score and other clinical covariates.

Results: Of the 208 lesions, $85.1 \%$ were diagnostic and $14.9 \%$ were non-diagnostic. The rates of non-diagnostic results were $8.9 \%, 25.5 \%$, and $57.1 \%$ for scores of $A, B$, and $C$, respectively. In the univariable analysis, scores of $B$ or $C$ were associated with a significantly higher rate of nondiagnostic results than scores of $A$ ( $58.1 \%$ vs. $24.9 \%, P<0.001)$. In the multivariable analysis, US visualization score of $B$ or $C$ (adjusted odds ratio [aOR], 2.7; $P=0.027$ ), high-risk needle pathway usage ( $a O R, 5.7 ; P=0.001)$, and lesion size $\leq 2.0 \mathrm{~cm}(a O R, 2.7 ; P=0.024)$ were independent risk factors for non-diagnostic results.

Conclusion: US-guided biopsy had a high diagnostic yield for focal hepatic lesions in patients at risk for HCC. US visualization score of B or C, lesion size $\leq 2.0 \mathrm{~cm}$, and high-risk needle pathway usage were independent risk factors for non-diagnostic results.

Keywords: Liver; Hepatocellular carcinoma; Ultrasonography; Biopsy; Diagnosis

\section{Introduction}

The Liver Imaging Reporting and Data System (LI-RADS) was introduced in 2011 [1] and was recently updated and fully integrated into the American Association for the Study of Liver Disease (AASLD) 2018 practice guideline to standardize the performance of liver imaging in patients at risk for

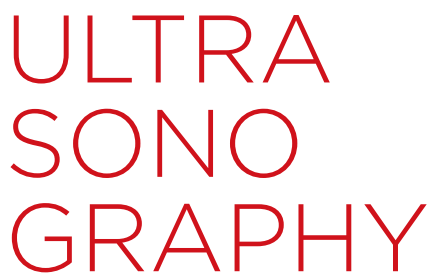

ORIGINAL ARTICLE

https://doi.org/10.14366/usg.19066 pISSN: 2288-5919 e elSSN: 2288-5943 Ultrasonography 2021;40:167-175

Received: November 1, 2019

Revised: May 7, 2020

Accepted: May 9, 2020

Correspondence to:

Sang Hyun Choi, MD, PhD, Department of Radiology and Research Institute of Radiology, Asan Medical Center, University of Ulsan College of Medicine, 88 Olympic-ro 43-gil, Songpa-gu, Seoul 05505, Korea

Tel. $+82-2-3010-1797$

Fax. +82-2-476-4719

E-mail: edwardchoi83@gmail.com

${ }^{*}$ Current affiliation: Department of Radiology, Hanyang University Guri Hospital, Hanyang University College of Medicine, Guri, Korea

This is an Open Access article distributed under the terms of the Creative Commons Attribution NonCommercial License (http://creativecommons.org/ licenses/by-nc/4.0/) which permits unrestricted noncommercial use distribution, and reproduction in any medium, provided the original work is properly cited.

Copyright (C) 2021 Korean Society of Ultrasound in Medicine (KSUM)

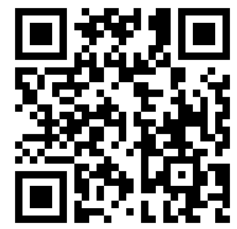

How to cite this article:

Kang JH, Choi SH, Kim SY, Lee SJ, Shin YM, Won HJ, et al. US LI-RADS visualization score: diagnostic outcome of ultrasoundguided focal hepatic lesion biopsy in patients at risk for hepatocellular carcinoma. Ultrasonography. 2021 Jan;40(1):167-175. 
hepatocellular carcinoma (HCC) [2]. The LI-RADS system categorizes each observation according to the relative likelihood of benignity and HCC (i.e., a system of classification ranging from LR-1 to LR-5) as well as differentiation from other malignancies (LR-M) $[3,4]$. As the LI-RADS classification is designed to maintain a high specificity for HCC, biopsy is usually unnecessary for confirmation of LR-5 lesions; however, biopsy may be considered for LR-4 or LR-M lesions, which have been shown to display features that are not typical of HCC on contrast-enhanced imaging [2].

According to the AASLD 2018 practice guideline, ultrasonography (US) is recommended as a surveillance tool in patients at risk for HCC $[2,5]$. The US LI-RADS system was developed to provide guidance regarding the performance, interpretation, and reporting of US examinations conducted for surveillance [6]. This algorithm includes a US visualization score $(A, B$, or $C)$ for use in assessing the quality and adequacy of the examination; additionally, it includes a detection score, such as the US LI-RADS category (US-1, US-2, or US-3) [6]. In addition to its use as a surveillance tool, US has been widely utilized for image-guided percutaneous liver biopsy [7], which is an effective method for achieving pathologic diagnosis of focal hepatic lesions with low mortality and morbidity and high diagnostic accuracy $[8,9]$. However, because biopsy is still associated with risks of bleeding and tumor seeding, as well as the possibility of a nondiagnostic result, routine biopsy is not suggested, and appropriate selection of patients is necessary. Given this background, it is important to be able to predict the likelihood of a non-diagnostic result and to understand the risk factors for non-diagnostic results in US-guided liver biopsy.

A recent study reported that a US visualization score of $C$ was associated with a higher false-negative rate for the detection of HCC than were scores of $A$ or $B$ ( $86 \%$ vs. $57 \%$ or $64 \%$, respectively) [10]. However, although the US visualization score was not developed to determine eligibility for focal liver biopsy, this score may have clinical utility in US-guided liver biopsy. We hypothesized that, as a measure of the quality and adequacy of the examination, the US visualization score may also provide useful information regarding the diagnostic outcome (that is, the production of either diagnostic or non-diagnostic results) of US-guided liver biopsy.

As such, the purpose of this study was to determine the diagnostic outcome of US-guided focal hepatic lesion biopsy in patients at risk for HCC and to evaluate the US visualization score as a risk factor for non-diagnostic results.

\section{Materials and Methods}

This retrospective study was approved by the institutional review board of our institution. The requirement for informed consent was waived because we retrospectively used data available in electronic medical records. However, informed consent for the biopsy procedure was obtained from all patients prior to biopsy.

\section{Patients}

A systematic computerized search of our hospital's database was performed to identify patients who underwent US-guided percutaneous core-needle biopsy of a hepatic lesion between January and December 2016. During this period, 720 US-guided percutaneous biopsies were performed in 688 patients. Of these 688 patients, 463 patients without risk factors for HCC were excluded. Of the remaining 225 patients at risk for HCC, 11 underwent a second biopsy because of a non-diagnostic result in the first biopsy; in these patients, the first biopsy result was included in this study, but the second biopsy result was excluded. In addition, six patients were excluded due to having non-focal infiltrative lesions. After all of these exclusion criteria were applied, 208 focal hepatic lesions in 208 patients were analyzed in this study (Fig. 1).

\section{US-Guided Biopsy Technique}

US examinations were performed using a LOGIQ E9 US system (General Electric Healthcare, Wauwatosa, WI, USA). Livers were studied at a low frequency of around $3.5 \mathrm{MHz}$ for general evaluation. The US examinations consisted of acquisitions of a series of images of both lobes of the liver for the evaluation of focal lesions, steatosis, and parenchymal echotexture. For focal hepatic lesions, the size, location, echogenicity, and vascularity of the nodules were evaluated.

Biopsies were performed by one of five abdominal radiologists, all of whom had more than 8 years of interventional US experience, or by a board-certified abdominal fellow under the supervision of a staff radiologist. Before biopsy, all patients met the coagulation profile requirements [11]: (1) platelet count $\geq 50,000 / \mathrm{mm}^{3}$, (2) an international normalized ratio of prothrombin time $\leq 1.4$, and (3) an activated partial thromboplastin time $\leq 35$ seconds. If a patient's platelet count was less than $50,000 / \mathrm{mm}^{3}$, transfusion was performed. All biopsies were performed with a 3.5- to 7-MHz transducer and a semiautomated biopsy gun with an 18-gauge needle (Stericut, TSK Laboratory, Tochigi, Japan). After induction of local anesthesia with $1 \%$ lidocaine at the needle puncture site, the end of the biopsy needle was advanced into the solid part of the nodule using a freehand technique. After the tip of the biopsy needle was advanced into the edge of the lesion, the vessels around the nodule were re-evaluated to minimize vessel injury. Onsite cytologic assessment was not available. The adequacy of tissue sampling was assessed according to the radiologic confidence on US images that the cutting needle had traversed the mass; additionally, if the lesion 
720 Liver biopsies in 688 patients between January and December 2016 1st exclusion:

- No risk factor of $\mathrm{HCC}$

(495 biopsies in 463 patients)
225 Liver biopsies in 225 patients at risk of $\mathrm{HCC}$

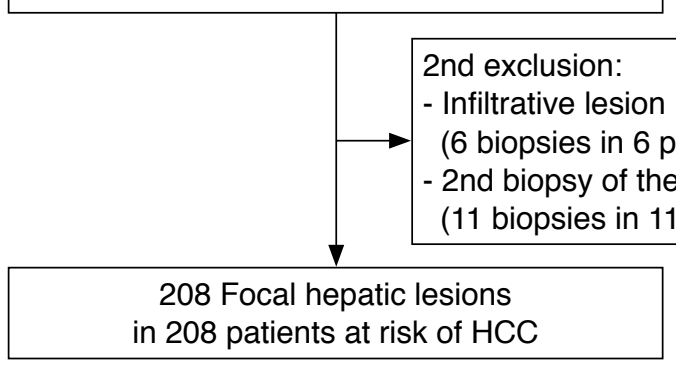

Fig. 1. Flowchart of the study population. HCC, hepatocellular carcinoma. was suspicious for a solid mass, adequacy was determined based on whether the gross specimen contained whitish tissue. Fusion imaging guidance was used to localize the index lesion accurately in cases of poor conspicuity. After the biopsy, the radiologists recorded the equipment used, the number of needle passages, and any complications encountered.

\section{Assessment of the US LI-RADS Visualization Score}

Two radiologists (each with $>6$ years of experience in hepatic imaging) who were blinded to any clinical information or final diagnoses independently reviewed the US images. Any discrepancies between the two readers were resolved by reaching a consensus at a joint re-evaluation after completion of the independent reviews.

According to the US LI-RADS version 2017, each US examination was assigned one of the following US visualization scores: (1) $A$, no or minimal limitations (limitations unlikely to meaningfully affect sensitivity); (2) B, moderate limitations (limitations that may obscure small masses); and (3) C, severe limitations (limitations that substantially lower the sensitivity for focal liver lesions) [6].

\section{Review of the Medical Records}

The medical records of the patients were reviewed by an author who was not involved in the assessment of US LI-RADS visualization scores. Using the pathology report, the diagnostic results of the biopsies were classified as malignant, benign, or non-diagnostic. A non-diagnostic result was assigned when non-lesional hepatic parenchyma was obtained on pathology due to a missed target and a definite tumor was found on subsequent repeat biopsy, surgery, or follow-up imaging.

The reviewer identified clinical characteristics (patient age, sex, body mass index [BMI], the presence of liver cirrhosis, and the specific risk factor for HCC) and US characteristics (lesion size, location, conspicuity, depth, degree of fatty liver, use of a highrisk needle pathway, and number of needle passages). The lesion location was defined according to the Couinaud classification. Lesion conspicuity was categorized as good, intermediate, or poor according to the degree of lesion margin clearly visible in the background liver parenchyma. If more than two-thirds of the lesion margin was clearly visible, the lesion conspicuity was considered to be good, but if less than one-third of the lesion margin was clearly visible, it was considered poor. Fatty liver disease was graded as follows: mild, increased liver echogenicity relative to that of the renal cortex; moderate, increased liver echogenicity with poor delineation of the echogenic walls of the portal vein; and severe, increased liver echogenicity with obscured diaphragmatic architecture [12]. Highrisk needle pathways were those in which a vessel was located 1-2 $\mathrm{mm}$ from the expected path or the index tumor was positioned $0-2$ $\mathrm{mm}$ from the ribs, lungs, or other organs [13].

\section{Statistical Analysis}

The study subjects were categorized into two groups according to the presence of diagnostic or non-diagnostic results. In order to evaluate the clinical importance of the US visualization score for US-guided biopsy, the frequency of each US visualization score was calculated for both the diagnostic and the non-diagnostic group and was then compared between the groups using the Pearson chisquare test or the Fisher exact test. The interobserver agreement for the US visualization score was determined using the kappa statistic.

To determine the risk factors for a non-diagnostic result, univariable analysis was performed using various clinical or US 
factors. Multivariable logistic regression analysis using a backward stepwise elimination method was then performed to identify independent factors associated with non-diagnostic results, with this analysis including the variables identified in the univariable analysis as being associated with a P-value of $<0.1$.

All statistical analyses were performed using SPSS version 21.0 (IBM Corp., Armonk, NY, USA), and $\mathrm{P}<0.05$ was considered to indicate a statistically significant difference.

\section{Results}

\section{Clinical and US Characteristics}

The clinical and US characteristics of the included patients and lesions are summarized in Table 1. The patients included 151 men (mean age, 60.2 years; range, 36 to 89 years) and 57 women (mean age, 60.8 years; range, 31 to 82 years). Of the 208 patients, 158 patients $(76.0 \%)$ had liver cirrhosis. The most common risk factor for HCC was hepatitis B $(n=129,62.0 \%)$ followed by alcoholic liver disease $(n=32,15.4 \%)$. Sixty-two patients $(29.8 \%)$ were overweight (BMI, $\left.25-30 \mathrm{~kg} / \mathrm{m}^{2}\right)$, and $16(7.7 \%)$ were obese (BMI, $>30 \mathrm{~kg} / \mathrm{m}^{2}$ ). Fifty-four patients (26.0\%) had fatty liver disease, with 47 having mild, six having moderate, and one having severe disease. Of the 208 lesions, $28.4 \%$ (59 or 208) were $\leq 2.0 \mathrm{~cm}$, and $12.0 \%$ (25 of 208) were biopsied using a high-risk needle pathway. Fusion imaging guidance using contrast-enhanced computed tomography or magnetic resonance imaging was utilized in $5.3 \%$ (11 of 208)

Table 1. Baseline characteristics of the patients, lesions, and procedures

\begin{tabular}{lc}
\hline \multicolumn{1}{c}{ Characteristic } & Value \\
\hline No. of patients & 208 \\
Age (y) & $60.4 \pm 10.7(31-89)$ \\
Sex & \\
Male & $151(72.6)$ \\
Female & $57(27.4)$ \\
Body weight $(\mathrm{kg})$ & $65.0 \pm 11.6(41.5-101.0)$ \\
BMl (kg/m $\left.{ }^{2}\right)$ & $24.1 \pm 3.7(15.5-39.1)$ \\
Liver cirrhosis & $158(76.0)$ \\
Risk factor & $129(62.0)$ \\
Hepatitis B & $16(7.7)$ \\
Hepatitis C & $1(0.5)$ \\
Hepatitis B and C & $32(15.4)$ \\
Alcoholic liver disease & $30(14.4)$ \\
Others ${ }^{\mathrm{a})}$ &
\end{tabular}

Table 1. Continued

\begin{tabular}{|c|c|}
\hline Characteristic & Value \\
\hline \multicolumn{2}{|l|}{ Fatty liver disease } \\
\hline None & $154(74.0)$ \\
\hline Mild & $47(22.6)$ \\
\hline Moderate & $6(2.9)$ \\
\hline Severe & $1(0.5)$ \\
\hline No. of lesions & 208 \\
\hline Size $(\mathrm{cm})$ & $4.2 \pm 3.1(1.0-19.0)$ \\
\hline$\leq 2.0$ & $59(28.4)$ \\
\hline$>2.0$ & $149(71.6)$ \\
\hline Depth (cm) & $5.8 \pm 1.9(2.4-12.0)$ \\
\hline$\leq 5.8^{b)}$ & $118(56.7)$ \\
\hline$>5.8$ & $90(43.3)$ \\
\hline \multicolumn{2}{|l|}{ US visualization score } \\
\hline A & $146(70.2)$ \\
\hline B & $55(26.4)$ \\
\hline C & $7(3.4)$ \\
\hline \multicolumn{2}{|l|}{ Lesion conspicuity } \\
\hline Good & $117(56.3)$ \\
\hline Intermediate & $79(38.0)$ \\
\hline Poor & $12(5.8)$ \\
\hline \multicolumn{2}{|l|}{ Location } \\
\hline Segment I & 0 \\
\hline Segment II & $10(4.8)$ \\
\hline Segment III & $17(8.2)$ \\
\hline Segment IV & $18(8.7)$ \\
\hline Segment V & $54(26.0)$ \\
\hline Segment VI & $35(16.8)$ \\
\hline Segment VII & $19(9.1)$ \\
\hline Segment VIII & $55(26.4)$ \\
\hline \multicolumn{2}{|l|}{ High-risk needle pathway } \\
\hline Yes & $25(12.0)$ \\
\hline No & $183(88.0)$ \\
\hline No. of needle passages & $2.4 \pm 0.5(1-5)$ \\
\hline$\leq 2$ & $133(63.9)$ \\
\hline$>2$ & $75(36.1)$ \\
\hline Use of fusion imaging guidance & $11(5.3)$ \\
\hline \multicolumn{2}{|l|}{ Diagnostic yield } \\
\hline Diagnostic & $177(85.1)$ \\
\hline Non-diagnostic & $31(14.9)$ \\
\hline \multicolumn{2}{|c|}{$\begin{array}{l}\text { Values are presented as mean } \pm \text { standard deviation (range) or number }(\%) \text {. } \\
\text { All of the needles used were 18-gauge. } \\
\text { BMI, body mass index; US, ultrasound. } \\
\text { alIncluded liver cirrhosis caused by nonalcoholic fatty liver disease }(n=9) \text {, recurrent } \\
\text { pyogenic cholangitis }(n=10) \text {, and cryptogenic liver cirrhosis }(n=11) .{ }^{b} \text { Mean value of } \\
\text { lesion depth for the total of } 208 \text { lesions. }\end{array}$} \\
\hline
\end{tabular}


lesions.

\section{Diagnostic Outcome of US-Guided Biopsy}

Of the 208 lesions in 208 patients, US-guided biopsy yielded diagnostic results in 177 (85.1\%) cases, while 31 (14.9\%) cases had non-diagnostic results. In the diagnostic group, malignancy was diagnosed in $83.7 \%$ of cases (149 of 177), including 89 HCCS (59.7\%), 31 cholangiocarcinomas (20.8\%), 13 metastases (8.7\%), seven combined HCC and cholangiocarcinomas $(4.7 \%)$, and nine other malignancies (neuroendocrine carcinoma, lymphoma, or sarcomatoid carcinoma). Benignity was diagnosed in $15.8 \%$ of cases (28 of 177) and included five dysplastic nodules, five hemangiomas, three cases of focal nodular hyperplasia, two inflammatory pseudotumors, one angiomyolipoma, and others (organizing abscess, eosinophilic abscess, parasitic granuloma, or scar tissue). In the nondiagnostic group, the final diagnoses were made via the surgical specimen $(n=4)$, additional biopsy $(n=11)$, or change in size on follow-up imaging $(n=16)$. The final diagnoses in the non-diagnostic group were $17 \mathrm{HCCs}(54.8 \%)$, four cholangiocarcinomas (12.9\%), two metastases (6.5\%), and eight benign lesions (25.8\%), including dysplastic nodules or organizing abscesses. The rates of diagnostic results determined by the five radiologists ranged from $82.2 \%$ (37 of 45 ) to $87.5 \%$ (42 of 48), without significant differences among these rates $(P=0.96)$ (Supplementary Table 1).

\section{US Visualization Score}

Of the total of 208 US-guided biopsies, 146 examinations (70.2\%) were assigned a US visualization score of $A, 55(26.4 \%)$ were scored as $B$, and seven $(3.4 \%)$ were scored as $C$. The rates of nondiagnostic results were $8.9 \%$ (13 of 146 ) for a score of $A, 25.5 \%$ (14 of 55 ) for a score of $B$, and $57.1 \%$ (4 of 7 ) for a score of $C$. The nondiagnostic result group had a significantly higher frequency of US visualization scores of $B$ or $C$ than the diagnostic result group (58.1\% [18 of 31 ] vs. $24.9 \%$ [ 44 of 177 ], $P<0.001$ ). The kappa value for the interobserver agreement in US visualization score was 0.6.

\section{Analysis of Risk Factors for a Non-diagnostic Biopsy Result}

The results of the univariable analysis are summarized in Table 2 . The risk factors displaying a significant difference between the diagnostic and non-diagnostic groups were a US visualization score of $B$ or $C(P<0.001)$, intermediate or poor lesion conspicuity $(P=0.004)$, use of a high-risk needle pathway $(P<0.001)$, lesion depth $>5.8 \mathrm{~cm}(P=0.028)$, and lesion size $\leq 2.0 \mathrm{~cm}(P=0.007)$. High BMI $\left(\geq 25 \mathrm{~kg} / \mathrm{m}^{2}\right)$ was more frequent in the non-diagnostic group than in the diagnostic group, but the difference did not reach statistical significance $(P=0.08)$. Other factors such as sex, the presence of liver cirrhosis, the type of risk factor, the degree
Table 2. Results of the univariable analysis used to determine the risk factors associated with non-diagnostic results

\begin{tabular}{|c|c|c|c|}
\hline Variable & $\begin{array}{c}\text { Diagnostic } \\
(n=177)\end{array}$ & $\begin{array}{c}\text { Non-diagnostic } \\
(n=31)\end{array}$ & P-value \\
\hline Sex & & & 0.826 \\
\hline Male & $129(85.4)$ & $22(14.6)$ & \\
\hline Female & $48(84.2)$ & $9(15.8)$ & \\
\hline $\mathrm{BMI}\left(\mathrm{kg} / \mathrm{m}^{2}\right)$ & & & 0.078 \\
\hline$<25$ & $115(88.5)$ & $15(11.5)$ & \\
\hline$\geq 25$ & $62(79.5)$ & $16(20.5)$ & \\
\hline Liver cirrhosis & & & 0.648 \\
\hline Present & $133(84.2)$ & $25(15.8)$ & \\
\hline Absent & $44(88.0)$ & $6(12.0)$ & \\
\hline Risk factor & & & 0.746 \\
\hline Viral hepatitis & $52(83.9)$ & $10(16.1)$ & \\
\hline Others & $125(85.6)$ & $21(14.4)$ & \\
\hline Fatty liver disease & & & 0.602 \\
\hline None or mild & $170(84.6)$ & $31(15.4)$ & \\
\hline Moderate or severe & $7(100)$ & 0 & \\
\hline Size $(\mathrm{cm})$ & & & 0.007 \\
\hline$\leq 2.0$ & $44(74.6)$ & $15(25.4)$ & \\
\hline$>2.0$ & $133(89.3)$ & $16(10.7)$ & \\
\hline Depth $(\mathrm{cm})$ & & & 0.028 \\
\hline$\leq 5.8$ & $106(89.8)$ & $12(10.2)$ & \\
\hline$>5.8$ & $71(78.9)$ & $19(21.1)$ & \\
\hline US visualization score & & & $<0.001$ \\
\hline$A$ & $133(91.1)$ & $13(8.9)$ & \\
\hline B or $C$ & $44(71.0)$ & $18(29.0)$ & \\
\hline Lesion conspicuity & & & 0.004 \\
\hline Good & $107(91.5)$ & $10(8.5)$ & \\
\hline Intermediate or poor & $70(76.9)$ & $21(23.1)$ & \\
\hline Location & & & 0.690 \\
\hline Segment I & 0 & 0 & \\
\hline Segment II & $9(90.0)$ & $1(10.0)$ & \\
\hline Segment III & $16(94.1)$ & $1(5.9)$ & \\
\hline Segment IV & $16(88.9)$ & $2(11.1)$ & \\
\hline Segment V & $46(85.2)$ & $8(14.8)$ & \\
\hline Segment VI & $31(88.6)$ & $4(11.4)$ & \\
\hline Segment VII & $16(84.2)$ & $3(15.8)$ & \\
\hline Segment VIII & $43(78.2)$ & $12(21.8)$ & \\
\hline High-risk needle pathway & & & $<0.001$ \\
\hline Yes & $14(56.0)$ & $11(44.0)$ & \\
\hline No & $163(89.1)$ & $20(10.9)$ & \\
\hline No. of needle passages & & & 0.942 \\
\hline$\leq 2$ & $113(85.0)$ & $20(15.0)$ & \\
\hline$>2$ & $64(85.3)$ & $11(14.7)$ & \\
\hline
\end{tabular}

Values are presented as number (\%).

BMI, body mass index; US, ultrasound. 
of fatty liver disease, the location of the lesion, and the number of needle passages showed no significant differences between the two groups.

In the multivariable logistic regression analysis (Table 3), a US visualization score of $\mathrm{B}$ or $\mathrm{C}$ (adjusted odds ratio [aOR], 2.7; 95\% confidence interval $[\mathrm{Cl}], 1.1$ to $6.3 ; \mathrm{P}=0.027$ ) (Fig. 2), the use of a high-risk needle pathway ( $\mathrm{aOR}, 5.7 ; 95 \% \mathrm{Cl}, 2.1$ to 15.8; $\mathrm{P}=0.001)$, and lesion size $\leq 2.0 \mathrm{~cm}(\mathrm{aOR}, 2.7 ; 95 \% \mathrm{Cl}, 1.1$ to $6.2 ; \mathrm{P}=0.024)$ were independent risk factors for a non-diagnostic result.

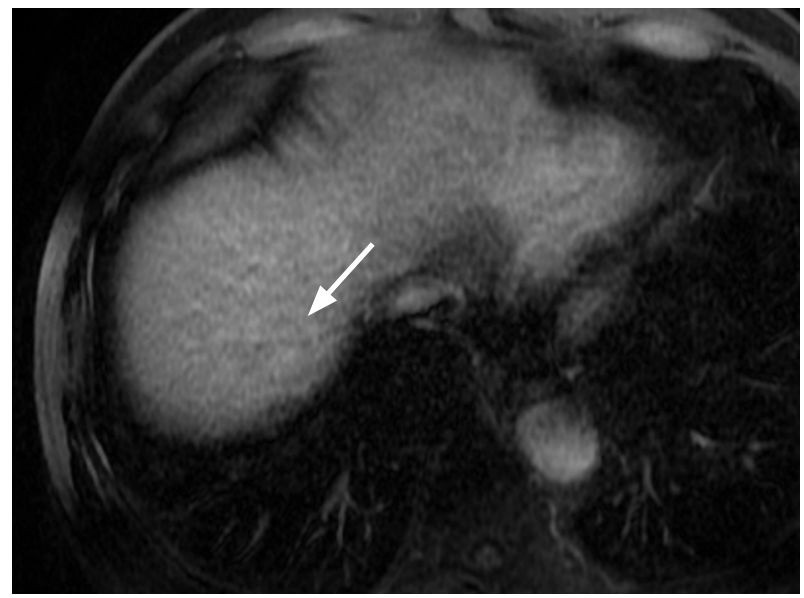

A

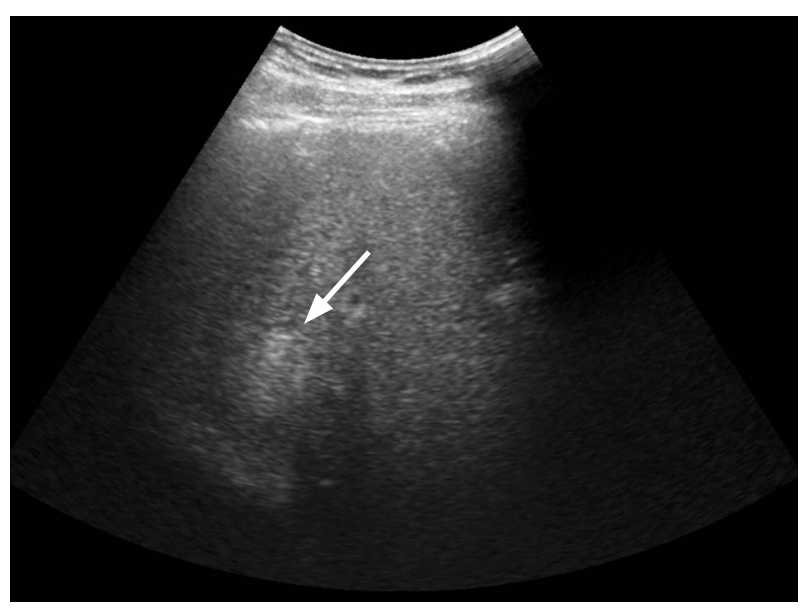

C
Table 3. Results of the multivariable logistic regression analysis used to determine the risk factors associated with nondiagnostic results

\begin{tabular}{lccc}
\hline \multicolumn{1}{c}{ Variable } & Adjusted OR & $95 \% \mathrm{Cl}$ & P-value \\
\hline BMI $\geq 25 \mathrm{~kg} / \mathrm{m}^{2}$ & 2.3 & $0.9-5.5$ & 0.057 \\
Size $\leq 2.0 \mathrm{~cm}$ & 2.7 & $1.1-6.2$ & 0.024 \\
Depth $>5.8 \mathrm{~cm}$ & 1.2 & $0.5-3.2$ & 0.697 \\
US visualization score B or C & 2.7 & $1.1-6.3$ & 0.027 \\
Intermediate or poor lesion & 1.8 & $0.7-4.4$ & 0.211 \\
conspicuity & & & 0.001 \\
High-risk needle pathway & 5.7 & $2.1-15.8$ &
\end{tabular}

$\mathrm{OR}$, odds ratio; $\mathrm{Cl}$, confidence interval; $\mathrm{BMI}$, body mass index; US, ultrasound.

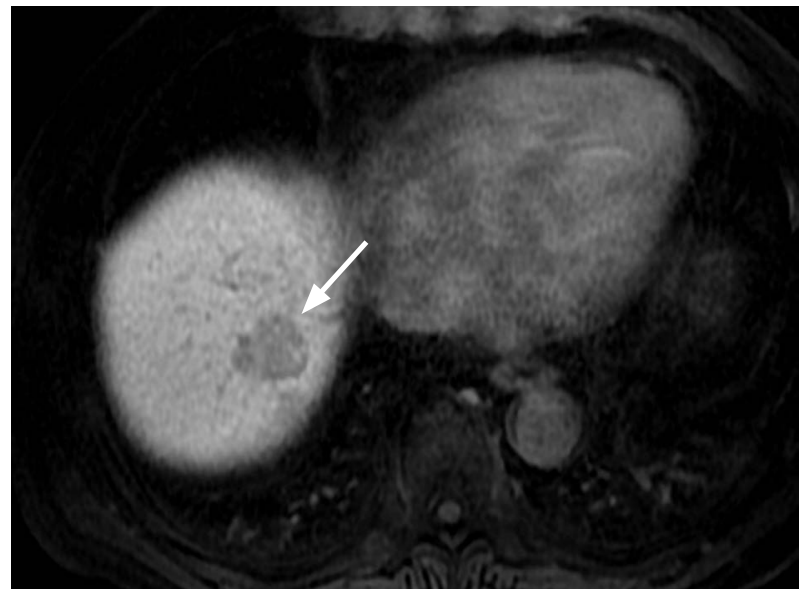

B

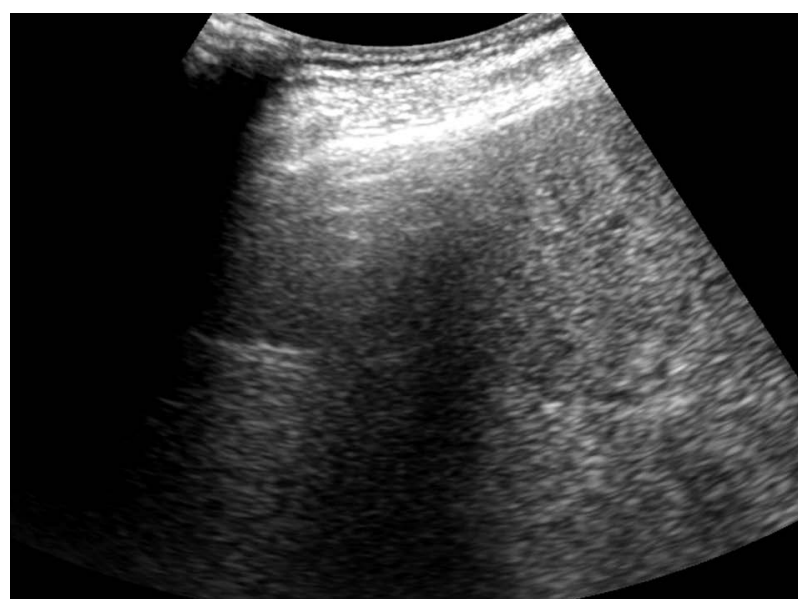

D

Fig. 2. A 68-year-old man with chronic hepatitis C.

A, B. Gadoxetate disodium-enhanced magnetic resonance imaging (MRI) shows a 23-mm nodule in hepatic segment VIII with no arterial phase hyperenhancement (arrow in A) and hepatobiliary-phase hypointensity (arrow in B). This nodule was categorized as Liver Imaging and Reporting Data System category 4 on MRI. C, D. On US, the corresponding nodule appears hyperechoic (arrow in C) with an indistinct margin. The US visualization score was assigned as $C$ because the background liver was moderately to severely heterogeneous, and the majority of the liver was not visualized (D). This nodule had a non-diagnostic pathologic result and was confirmed as hepatocellular carcinoma after surgical resection. 


\section{Discussion}

This study demonstrated that US-guided liver biopsy resulted in a high diagnostic yield (85.1\% [177 of 208]) for focal hepatic lesions in patients at risk for HCC. A US visualization score of B or C was noted more frequently in the group of patients with non-diagnostic results than in the group with diagnostic results (58.1\% [18 of 31 ] vs. $24.9 \%$ [44 of 177 ], respectively; $P<0.001$ ). In addition, a US visualization score of $\mathrm{B}$ or $\mathrm{C}(\mathrm{aOR}, 2.7 ; 95 \% \mathrm{Cl}, 1.1$ to 6.3 ; $\mathrm{P}=0.027)$, the use of a high-risk needle pathway (aOR, $5.7 ; 95 \%$ $\mathrm{Cl}, 2.1$ to 15.8; $\mathrm{P}=0.001)$, and lesion size $\leq 2.0 \mathrm{~cm}(\mathrm{aOR}, 2.7 ; 95 \%$ $\mathrm{Cl}, 1.1$ to $6.2 ; \mathrm{P}=0.024$ ) were determined to be independent risk factors for a non-diagnostic result.

In this study, the rate of diagnostic results was $85.1 \%$ (177 of 208), which is slightly lower than the rates found in several recent studies $(93.3 \%-98.6 \%)$ [7-9]. This discrepancy might be explained by differences in the study subjects, in that the present study included only patients at risk for HCC, such as those with liver cirrhosis, while the other studies were not limited to patients at risk for HCC. Cirrhotic liver is thought to be an obstacle for the biopsy of focal liver lesions because of the inherent heterogeneity of the liver parenchyma and the nodular liver morphology $[9,14]$. When the background liver parenchyma is taken into consideration, the rate of diagnostic results obtained in this study is similar to those found in previous studies $(87.0 \%-87.6 \%)$ that focused on patients or subgroups of patients with liver cirrhosis $[9,14]$.

The US visualization score reflects factors that may affect liver visualization or nodule detection [6]. Recent studies have reported that a US visualization score of $C$ was associated with an $86 \%$ false-negative rate for the diagnosis of HCC in surveillance US examinations [10], and parenchymal macronodularity may hinder the detection of early-stage tumors during US surveillance [15]. In this study, a higher rate of non-diagnostic results was associated with a US visualization score of $B$ or $C$ than with a score of $A$, and a US visualization score of $B$ or $C$ was determined to be an independent risk factor for a non-diagnostic result on US-guided liver biopsy. This result appears to align with an earlier study that reported lesion conspicuity to be a significant factor associated with successful liver biopsy [16]. In other words, given that low lesion conspicuity may obscure small lesions in the case of heterogeneous liver background parenchyma $[9,14]$, a US visualization score of $B$ or $C$ is a risk factor for a non-diagnostic result on US-guided liver biopsy. However, because the lesion conspicuity is not influenced only by US visualization score but also by lesion characteristics, these factors should be considered together.

The sensitivity of liver biopsy-based diagnosis of HCC may depend on various factors, including lesion location, size, and the expertise of both the person performing the biopsy and the pathologist [17]. In this study, the use of a high-risk needle pathway was one of the most important risk factors for a non-diagnostic result. Because the use of such a high-risk needle pathway could not secure sufficient distance between the index tumor and major vascular or biliary structures [13], the biopsy needle could not be advanced deeply, yielding in a non-diagnostic result. Therefore, planning the biopsy needle pathway is important to improve diagnostic outcomes as well as to minimize complications. In addition, lesion size $\leq 2.0 \mathrm{~cm}$ was another independent risk factor for a non-diagnostic result. A previous prospective study also reported a very similar result, with a false-negative result rate of $30 \%$ in lesions smaller than $2.0 \mathrm{~cm}$ at the first liver biopsy [18]. Making a pathologic diagnosis of lesions smaller than $2.0 \mathrm{~cm}$ is generally more challenging than doing so for larger lesions because these smaller lesions often represent well-differentiated tumors [19]. However, as previous studies have reported conflicting results regarding lesion size as a risk factor for a non-diagnostic result-with some reporting a decreased diagnostic yield with a smaller lesion size $[14,20]$, and others reporting no significant difference in diagnostic yield according to lesion size $[8,9,14]$-further studies are needed to determine whether lesion size is a risk factor for a non-diagnostic result on US-guided liver biopsy.

This study had several limitations. First, the retrospective nature of the study design may have led to selection bias. During the study period, eight biopsies were canceled at the time of the procedure due to poor visibility of the index lesion or the requirement of a high-risk needle pathway for liver biopsy. Notably, as patients with poor lesion conspicuity or a US visualization score of $C$ could be excluded at the referral stage, the number of patients with these parameters may be underestimated. Also, as liver biopsies were usually performed in indeterminate cases such as LR-4 or LR-M lesions, a higher proportion of malignant lesions than benign lesions was noted. Second, we retrospectively assigned the US visualization score using previous US images captured th the time of biopsy. This assessment was inevitable because our study subjects were examined in 2016, before the 2017 version of the US LIRADS system was introduced. To minimize this limitation, the two readers independently reviewed the images and then reached a joint consensus in the case of any discrepancies. In addition, as the US visualization scoring system was developed for US examinations performed for screening or surveillance and limited images were available for US-guided biopsy, the use of the US visualization score in US-guided liver biopsy may differ from its use in US surveillance. Further studies are needed to develop US visualization scores specifically for US-guided liver biopsy.

In conclusion, US-guided liver biopsy resulted in a high diagnostic 
yield for focal hepatic lesions in patients at risk for HCC. A higher rate of non-diagnostic results was noted with a US visualization score of $B$ or $C$ than with a score of $A$, and a US visualization score of $B$ or $C$, in addition to a lesion size $\leq 2.0 \mathrm{~cm}$ and the use of a highrisk needle pathway, was found to be an independent risk factor for a non-diagnostic result on US-guided liver biopsy. US visualization score may be clinically useful in the planning of US-guided liver biopsy in patients at risk for HCC.

ORCID: Ji Hun Kang: https://orcid.org/0000-0002-4213-9428; Sang Hyun Choi: https://orcid.org/0000-0002-6898-6617; So Yeon Kim: https://orcid.org/0000-00016853-8577; So Jung Lee: https://orcid.org/0000-0003-3269-0141; Yong Moon Shin: https://orcid.org/0000-0001-7165-3154; Hyung Jin Won: https://orcid.org/00000002-0704-4239; Pyo-Nyun Kim: https://orcid.org/0000-0002-3073-0288

\section{Author Contributions}

Conceptualization: Choi SH. Data acquisition: Kim SY, Lee SJ, Shin YM, Won HJ, Kim PN. Data analysis or interpretation: Kang JH, Choi SH. Drafting of the manuscript: Kang JH. Choi SH. Critical revision of the manuscript: Kim SY. Approval of the final version of the manuscript: all authors

\section{Conflict of Interest}

No potential conflict of interest relevant to this article was reported.

\section{Acknowledgments}

This work was supported by a National Research Foundation of Korea (NRF) grant funded by the Korean government (MSIT) (grant number: NRF-2019R1G1A1099743).

\section{Supplementary Material}

Supplementary Table 1. Diagnostic outcomes of ultrasound-guided liver biopsy performed by five individual radiologists (https://doi. org/10.14366/usg.19066).

\section{References}

1. Mitchell DG, Bruix J, Sherman M, Sirlin CB. LI-RADS (Liver Imaging Reporting and Data System): summary, discussion, and consensus of the LI-RADS Management Working Group and future directions. Hepatology 2015;61:1056-1065.

2. Marrero JA, Kulik LM, Sirlin CB, Zhu AX, Finn RS, Abecassis MM, et al. Diagnosis, staging, and management of hepatocellular carcinoma: 2018 practice guidance by the American Association for the Study of Liver Diseases. Hepatology 2018;68:723-750.

3. Chernyak V, Fowler KJ, Kamaya A, Kielar AZ, Elsayes KM, Bashir MR, et al. Liver Imaging Reporting and Data System (LI-RADS) version
2018: imaging of hepatocellular carcinoma in at-risk patients. Radiology 2018;289:816-830.

4. Kim TH, Kim SY, Tang A, Lee JM. Comparison of international guidelines for noninvasive diagnosis of hepatocellular carcinoma: 2018 update. Clin Mol Hepatol 2019;25:245-263.

5. Purcell Y, Copin P, Paulatto L, Pommier R, Vilgrain V, Ronot M. Hepatocellular carcinoma surveillance: Eastern and Western perspectives. Ultrasonography 2019;38:191-199.

6. Morgan TA, Maturen KE, Dahiya N, Sun MR, Kamaya A; American College of Radiology Ultrasound Liver Imaging and Reporting Data System (US LI-RADS) Working Group. US LI-RADS: ultrasound liver imaging reporting and data system for screening and surveillance of hepatocellular carcinoma. Abdom Radiol (NY) 2018;43:41-55.

7. Howlett DC, Drinkwater KJ, Lawrence D, Barter S, Nicholson T. Findings of the UK national audit evaluating image-guided or image-assisted liver biopsy. Part I. Procedural aspects, diagnostic adequacy, and accuracy. Radiology 2012;265:819-831.

8. Appelbaum L, Kane RA, Kruskal JB, Romero J, Sosna J. Focal hepatic lesions: US-guided biopsy: lessons from review of cytologic and pathologic examination results. Radiology 2009;250:453-458.

9. Ma X, Arellano RS, Gervais DA, Hahn PF, Mueller PR, Sahani DV. Success of image-guided biopsy for small $(\leq 3 \mathrm{~cm})$ focal liver lesions in cirrhotic and noncirrhotic individuals. J Vasc Interv Radio 2010;21:1539-1547.

10. Son JH, Choi SH, Kim SY, Jang HY, Byun JH, Won HJ, et al. Validation of US Liver Imaging Reporting and Data System version 2017 in patients at high risk for hepatocellular carcinoma. Radiology 2019:292:390-397.

11. Patel IJ, Davidson JC, Nikolic B, Salazar GM, Schwartzberg MS, Walker TG, et al. Consensus guidelines for periprocedural management of coagulation status and hemostasis risk in percutaneous image-guided interventions. J Vasc Interv Radiol 2012;23:727-736.

12. Saadeh S, Younossi ZM, Remer EM, Gramlich T, Ong JP, Hurley M, et al. The utility of radiological imaging in nonalcoholic fatty liver disease. Gastroenterology 2002;123:745-750.

13. Kim YS, Park MJ, Rhim H, Lim HK. Technical tips for successful USguided percutaneous RF ablation of the hepatic tumor [Internet]. Vienna: European Society of Radiology, 2010 [cited 2020 Feb 26]. Available from: https://epos.myesr.org/poster/esr/ecr2010/C-1951.

14. Caturelli E, Solmi L, Anti M, Fusilli S, Roselli P, Andriulli A, et al. Ultrasound guided fine needle biopsy of early hepatocellular carcinoma complicating liver cirrhosis: a multicentre study. Gut 2004;53:1356-1362.

15. Kim YY, An C, Kim DY, Aljogiman KS, Choi JY, Kim MJ. Failure of hepatocellular carcinoma surveillance: inadequate echogenic window and macronodular parenchyma as potential culprits. Ultrasonography 2019;38:311-320.

16. Kim JS, Won HJ, Lee SJ, Kim SY, Shin YM, Kim PN. Utility and safety 
of repeated ultrasound-guided core needle biopsy of focal liver masses. J Ultrasound Med 2018;37:447-452.

17. European Association for the Study of the Liver. EASL clinical practice guidelines: management of hepatocellular carcinoma. J Hepatol 2018;69:182-236.

18. Forner A, Vilana R, Ayuso C, Bianchi L, Sole M, Ayuso JR, et al. Diagnosis of hepatic nodules $20 \mathrm{~mm}$ or smaller in cirrhosis: Prospective validation of the noninvasive diagnostic criteria for hepatocellular carcinoma. Hepatology 2008;47:97-104.

19. Roskams T, Kojiro M. Pathology of early hepatocellular carcinoma: conventional and molecular diagnosis. Semin Liver Dis 2010;30:1725.

20. Reading CC, Charboneau JW, James EM, Hurt MR. Sonographically guided percutaneous biopsy of small (3 $\mathrm{cm}$ or less) masses. AJR Am J Roentgenol 1988;151:189-192. 